\title{
Article
}

\section{Control Structure Selection and Performance Assessment for Disturbance Rejection in MIMO Processes}

Hsiao-Ping Huang, Feng-Yi Lin, and Jyh-Cheng Jeng

Ind. Eng. Chem. Res., 2007, 46 (26), 9170-9178 • DOI: 10.1021/ie070382i

Downloaded from http://pubs.acs.org on November 18, 2008

\section{More About This Article}

Additional resources and features associated with this article are available within the HTML version:

- $\quad$ Supporting Information

- $\quad$ Access to high resolution figures

- $\quad$ Links to articles and content related to this article

- $\quad$ Copyright permission to reproduce figures and/or text from this article

\section{View the Full Text HTML}

\section{ACS Publications}




\title{
Control Structure Selection and Performance Assessment for Disturbance Rejection in MIMO Processes
}

\author{
Hsiao-Ping Huang,* Feng-Yi Lin, and Jyh-Cheng Jeng \\ Department of Chemical Engineering, National Taiwan University, Taipei 106-17, Taiwan
}

\begin{abstract}
A decoupling multivariable controller (DMVC) is generally considered more general than a multiloop controller (MLC). This is because a DMVC can eliminate the interactions in an MIMO process and result in better performance. Nevertheless, MLC may perform better than the DMVC while dealing with disturbance rejection. If the control objective is aimed at disturbance rejection, selection between these two types of controllers for better control is thus necessary. In this article, a method to assess the performances of these two types of controllers for a given process is proposed. Given an MIMO transfer-function model, the achievable performance is derived; indices for assessing these two systems are proposed. By making use of these indices, a proper type of system (DMVC or MLC) can be selected in the precedence of designing the controller.
\end{abstract}

\section{Introduction}

The multiloop controllers (MLC) and decoupling multivariable controllers (DMVC) have been used in the control of MIMO chemical plants. The MLC has a diagonal structure with PID controllers on the diagonal entries. On the other hand, the DMVC has a full-matrix structure. Because of the structural difference, it is usually considered that DMVC is superior to the MLC in control. However, this is not quite true. In an article by Niederlinski, ${ }^{1}$ an MLC was illustrated to have better load rejection ability than a DMVC does. In general, the DMVC is aimed to inverse the transfer-function matrix (TFM) of the process, $G_{\mathrm{p}}$, so that the loop transfer function (LTF) becomes diagonal or diagonal dominant. In other words, most multivariable controllers embed, implicitly or explicitly, the inverse of $G_{\mathrm{p}}$ in their designs. Because of this requirement for $G_{\mathrm{p}}$ inversion, DMVC may not perform better than MLC. In contrast, the MLC may suffer from the interactions in a multi-input-multioutput (MIMO) process, which may degrade the performance seriously. On many occasions, depending on the process TFM, the DMVC has no absolute advantage over the MLC in performance, especially in dealing with disturbance rejection. To achieve better control performance, guidance for the selection of the control structure prior to the design of the controller will be helpful.

For the selection of controller structure, theoretically achievable performances of these two types of controllers in MIMO control systems are required. In fact, the issue of theoretically achievable performance has been extensively studied. Articles in the literature and in books ${ }^{2-5}$ are available. In the past, the minimum variance was benchmarked as the optimal achievable performance index. Although theoretically best, this benchmarked performance is not practical because the performance that a practical system can achieve is far off from that. In fact, control performance of an MIMO system is limited not only by the method of control, but also by the modeling and realization of the controller.

In this article, an attempt to provide guidance for controlstructure selection prior to controller design is motivated. The systems consisting of either MLC or $\mathrm{DMVC}^{6-8}$ and a unity

* To whom correspondence should be addressed. Tel: $886-2$ 23638999. Fax: 886-2-2362-3935. E-mail: huanghpc@ntu.edu.tw (H-P.H.).F90524013@ntu.edu.tw (F-Y.L.).jcjeng@ntu.edu.tw (J-C.J.).
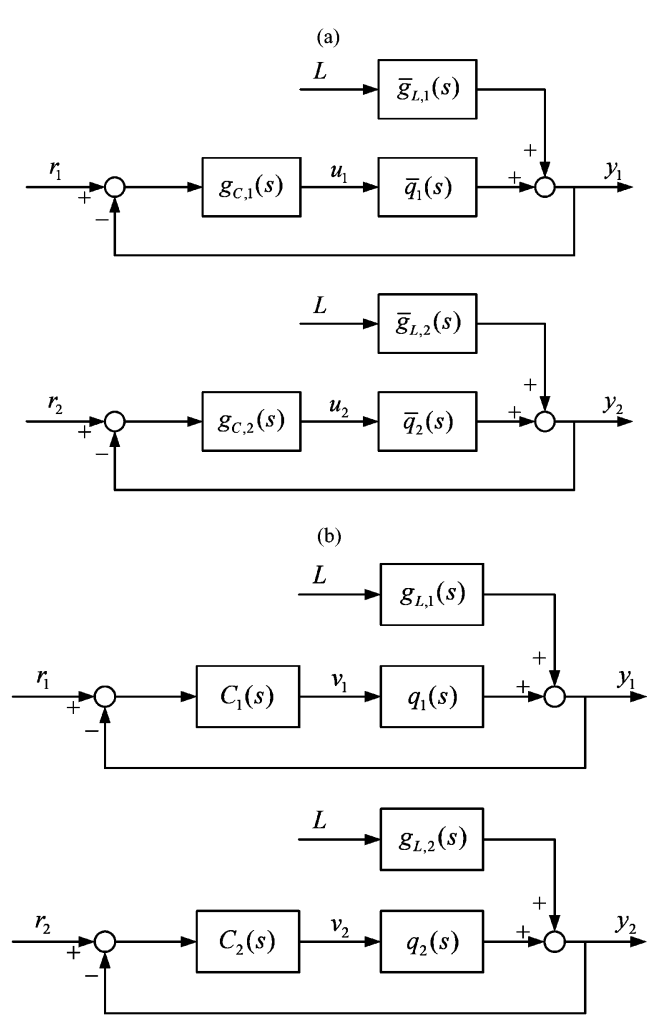

Figure 1. Equivalent single-loop block diagrams of a $2 \times 2$ process (a) MLC (b) DMVC.

feedback will be the scope to be considered. As we have mentioned earlier, a DMVC system considered here aims to have an LTF being diagonal or diagonally dominant. In contrast, the LTF matrix of an MLC system will be a full matrix. The main focus of the performance will be on the capability of the systems in disturbance rejection. The achievable performance for this purpose using each of the controllers will be explored. Indices as criteria to select the controller structure are proposed. Validation of the effectiveness of the presented indices is also illustrated with examples.

\section{Illustrative Comparison of MLC and DMVC Systems}

Here, the fact that DMVC does not always perform better than MLC in the control will be illustrated with three examples. 
Let an MIMO process be given as the following:

$$
Y(s)=G_{\mathrm{p}}(s) U(s)+G_{\mathrm{L}}(s) L(s)
$$

where $Y \in R^{n \times 1}$ is the output vector, $U \in R^{n \times 1}$ is the manipulated input, $L \in R^{1 \times 1}$ is the load input, and $G_{\mathrm{p}}(s)$ and $G_{\mathrm{L}}(s)$ are the transfer-function matrices (TFMs). To simplify the indexing notations used in the following text, the notation $G_{\mathrm{p}}(s)$ will simply be denoted as $G(s)$, later on.

An MLC system is conceptually considered to behave dynamically as decentralized loops. For example, a $2 \times 2$ system is decomposed into two equivalent single-loop systems as shown in part (a) of Figure 1. The equivalent processes, $\bar{q}_{1}(s)$ and $\bar{q}_{2^{-}}$ $(s)$ in a $2 \times 2$ MIMO control system, can be found elsewhere. ${ }^{9,10}$ The equivalent load TFM can be written as

$$
\begin{aligned}
& \bar{g}_{\mathrm{L}, 1}=g_{\mathrm{L}, 1}-g_{12} g_{22}{ }^{-1} g_{\mathrm{L}, 2} h_{2} \\
& \bar{g}_{\mathrm{L}, 2}=g_{\mathrm{L}, 2}-g_{21} g_{11}{ }^{-1} g_{\mathrm{L}, 1} h_{1}
\end{aligned}
$$

with

$$
h_{\mathrm{i}}=\frac{g_{\mathrm{C}, i} g_{i i}}{1+g_{\mathrm{C}, i} g_{i i}}, i=1,2
$$

and $g_{\mathrm{C}, i}$ is the controller of $i$ th loop.

In general, a higher dimensional system can be formulated in the same way. To do so, the matrices are first permuted and partitioned into the following forms:

$G^{(i)}=\left[\begin{array}{ll}g_{i i} & G_{12}^{(i)} \\ G_{21}^{(i)} & G_{22}^{(i)}\end{array}\right] ; G_{\mathrm{C}}^{(i)}=\left[\begin{array}{ll}g_{\mathrm{C}, i} & 0 \\ 0 & G_{\mathrm{C}, 2}^{(i)}\end{array}\right] ; G_{\mathrm{L}}^{(i)}=\left[\begin{array}{l}g_{\mathrm{L}, i} \\ G_{\mathrm{L}, 2}^{(i)}\end{array}\right]$

Then, the equivalent load and open-loop transfer functions of the $i$ th equivalent system are given as

$$
\begin{aligned}
\bar{g}_{\mathrm{L}, i} & =g_{\mathrm{L}, i}-G_{12}^{(i)}\left[G_{22}^{(i)}\right]^{-1}\left\{I-\left(I+G_{22}^{(i)} G_{\mathrm{C}, 2}^{(i)}\right)^{-1}\right\} G_{\mathrm{L}, 2}^{(i)} \\
\bar{q}_{i} & =g_{i i}-G_{12}^{(i)}\left[G_{22}^{(i)}\right]^{-1}\left\{I-\left(I+G_{22}^{(i)} G_{\mathrm{C}, 2}^{(i)}\right)^{-1}\right\} G_{21}^{(i)}
\end{aligned}
$$

The controllers may then be synthesized by using either of the following methods:

1. Detuning methods ${ }^{11,12}$

2. Sequential loop closing methods ${ }^{13-16}$

3. Iterative or trial-and-error methods ${ }^{17,18}$

4. Simultaneous equation solving or optimization methods ${ }^{19,20}$

5. Independent methods ${ }^{21-24}$

Providing controllers in all of the other loops, the transfer functions $\bar{q}_{i}$ and $\bar{g}_{\mathrm{L}, i}$ can be found and the performance of each loop can be evaluated. Because these two transfer functions in eq 5 and eq 6 depend on all of the controllers in other loops, the designs of controllers are usually coupled and are conducted in an iterative manner.

A DMVC, denoted as $K(s)$, consists of a decoupler $D(s)$ and a filter matrix $C(s)$. As in the design of Huang and Lin, ${ }^{8}$ it is possible to find a decoupled process $Q(s)$, as shown in part (b) of Figure 1 for a $2 \times 2$ system. Methods in the literature may lead to a different $Q(s)$, which leads to different single-loop based regulation problems. After having this decoupled $Q(s)$, a diagonal matrix $C(s)$ is designed by making use of methods for SISO systems.

For comparison purposes, the MLC and DMVC in the following illustrations are designed according to the methods as follows.



Figure 2. Assessment of the performance in the disturbance rejection for case 1.

(1) For MLC, the diagonal entries are limited to have a PID controller. A unit step of $L$ is then used to test for their performances. Parameters of the controller in the MLC are computed by directly searching for minimal ISE in a sequential and iterative loop closing manner until a total minimum of ISE is reached.

(2) For DMVC, the method of Huang and $\mathrm{Lin}^{8}$ is used. First, $G(s)$ is factorized into

$$
G(s)=e^{-\Theta s} G_{\mathrm{o}}(s)
$$

where $\Theta=\operatorname{diag}\left[\theta_{i i}^{*} ; i=1,2, \ldots, n\right]$ and $\theta_{i i}^{*}$ is the shortest dead time of $i$ th row of $G(s)$. A decoupler is given by post multiplying the adjoint of $G_{0}(s)$ by a diagonal matrix $Z(s)$, which makes the decoupler proper, that is

$$
D(s)=\operatorname{Adj}\left[G_{\mathrm{o}}(s)\right] Z(s)
$$

An additional purpose of the matrix $Z(s)$ is to speed up the dynamics of the decoupled open-loop process, which is given as

$$
Q(s)=G(s) D(s)=\left|G_{\mathrm{o}}(s)\right| e^{-\Theta s} Z(s)
$$

A diagonal $C(s)$ is then designed for $Q(s)$ to achieve a minimal ISE in responding to a unit-step $L$ input.

By making use of the above-mentioned methods to design the MLC and DMVC, the performances of the controllers will be compared, using example processes in the following:

Case 1. Consider the following transfer-function matrices for the process and load.

$G(s)=\left[\begin{array}{cc}\frac{7 e^{-5 s}}{10 s+1} & \frac{-3 e^{-5 s}}{20 s+1} \\ \frac{4 e^{-10 s}}{10 s+1} & \frac{-6 e^{-10 s}}{20 s+1}\end{array}\right]$ and $G_{\mathrm{L}}(s)=\left[\begin{array}{l}\frac{k_{\mathrm{L}, 1} e^{-5 s}}{30 s+1} \\ \frac{k_{\mathrm{L}, 2} e^{-10 s}}{30 s+1}\end{array}\right]$

The performances of the systems have been tested for disturbance rejection along different directions in the $k_{\mathrm{L}, 1}$ versus $k_{\mathrm{L}, 2}$ plane. For each pair of $k_{\mathrm{L}, 1}$ and $k_{\mathrm{L}, 2}$, the optimal performances in terms of ISE values of MLC and DMVC can be found and compared. As shown in Figure 2 , the $k_{\mathrm{L}, 1}$ versus $k_{\mathrm{L}, 2}$ plane can be divided into four areas, where the dark areas mean that MLC has a smaller ISE than DMVC does. For example, when $k_{\mathrm{L}, 1}=5$ and $k_{\mathrm{L}, 2}=4$, the responses and ISE obtained from these two control systems are given in Figure 3. 

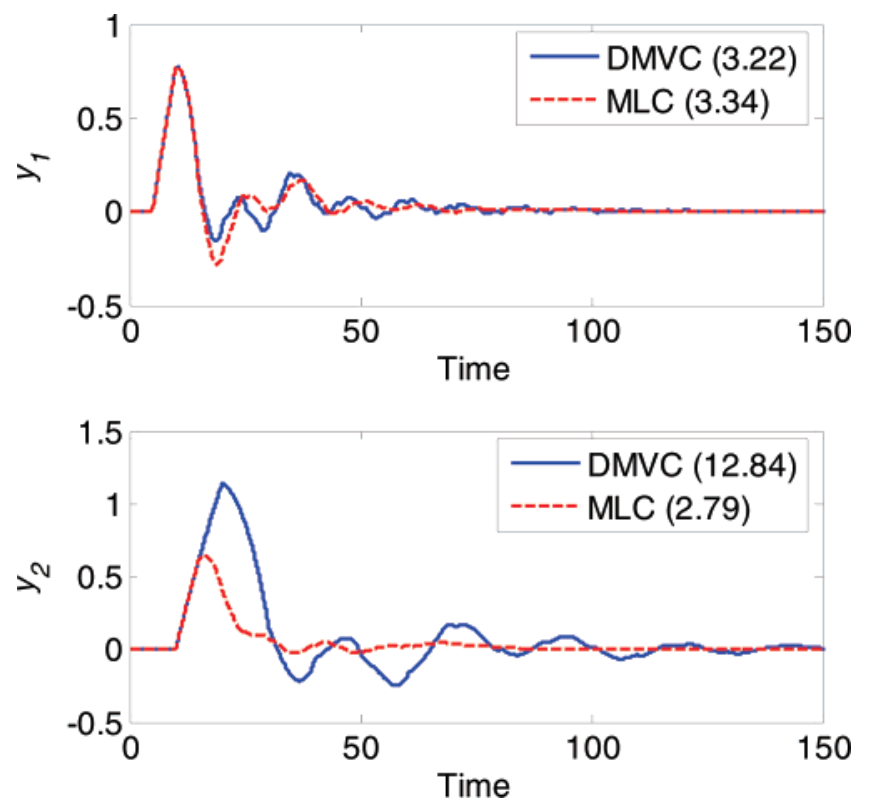

Figure 3. Responses and ISE values in case 1 with $k_{\mathrm{L}, 1}=5$ and $k_{\mathrm{L}, 2}=4$.
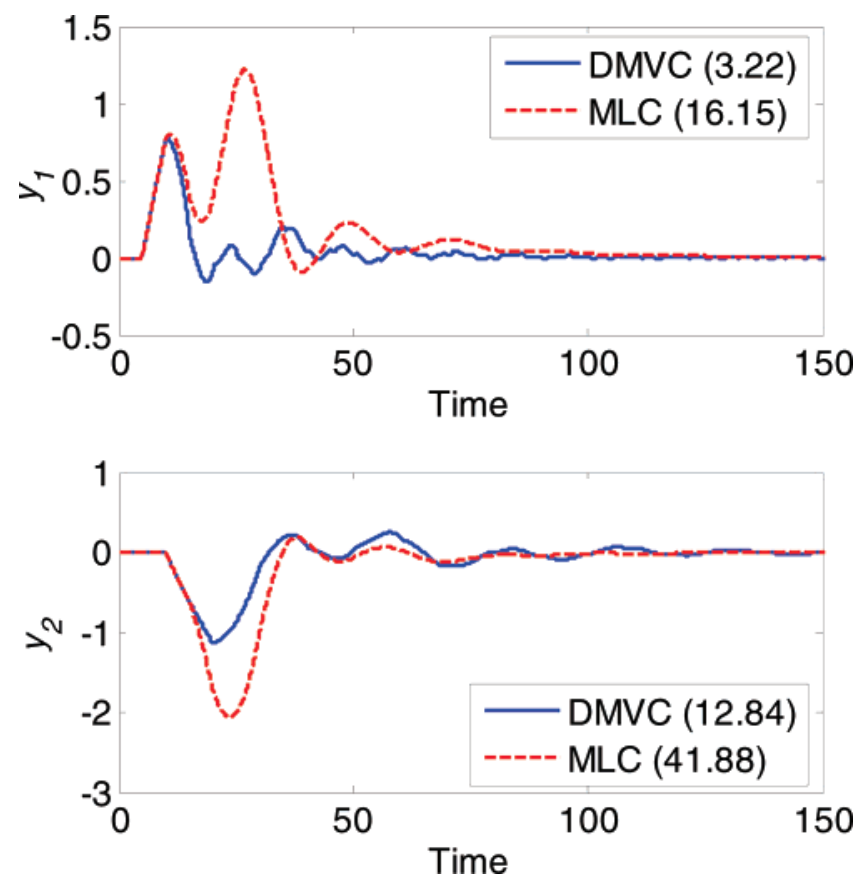

Figure 4. Responses and ISE values in case 1 with $k_{\mathrm{L}, 1}=5$ and $k_{\mathrm{L}, 2}=$ -4 .

In contrast, reverse the sign of $k_{\mathrm{L}, 2}$, that is $k_{\mathrm{L}, 2}=-4$, then, the point $\left(k_{\mathrm{L}, 1}, k_{\mathrm{L}, 2}\right)$ lies in the gray area of Figure 2 . As shown by the simulated responses in Figure 4, the performances of DMVC are better than those of the MLC, even though the openloop processes in the two cases are the same. From these results, it is found that the DMVC is not always superior to the MLC (e.g., the dark zone in Figure 2).

Case 2. Consider the following transfer-function matrices.

$G(s)=\left[\begin{array}{cc}\frac{9 e^{-5 s}}{10 s+1} & \frac{0.8 e^{-10 s}}{20 s+1} \\ \frac{0.9 e^{-5 s}}{10 s+1} & \frac{8 e^{-10 s}}{20 s+1}\end{array}\right]$ and $G_{\mathrm{L}}(s)=\left[\begin{array}{c}\frac{5 e^{-5 s}}{30 s+1} \\ \frac{3 e^{-10 s}}{30 s+1}\end{array}\right]$

With the same approaches of design, the control responses are obtained and compared in Figure 5. Notice that the inversion
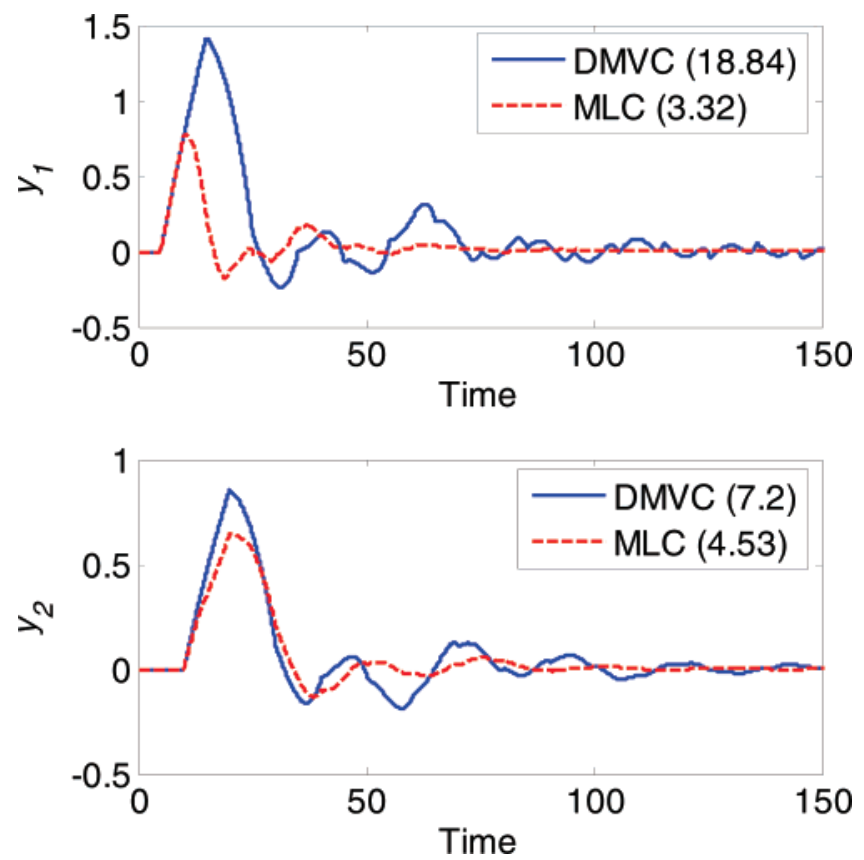

Figure 5. Responses and ISE values in case 2.

of $G(s)$ for designing DMVC requires extra dead time to make the inversion feasible. This causes the first output of the system to be retarded.

From the above examples, a properly chosen controller type is important to achieve better control performances.

\section{Achievable Performance}

In a single-loop case, the closed-loop responses for disturbance rejection can be derived as

$$
y(s)=g_{\mathrm{L}}(s)[1-h(s)] L(s)=y_{\mathrm{L}, 1}(s)-y_{\mathrm{L}, 2}(s)
$$

where $g_{\mathrm{L}}(s)$ is the disturbance transfer function and $h(s)$ is the complementary sensitivity function. The result in eq 12 means that the disturbance $y_{\mathrm{L}, 1}(s)$ is compensated by a reverse response $y_{\mathrm{L}, 2}(s)$, which is generated by the feedback control loop. The achievable responses for disturbance rejection can be found by designing $y_{\mathrm{L}, 2}(s)$ to achieve the performance requirement. For example, Huang and $\operatorname{Lin}^{8}$ find $h(s)$ and $y_{\mathrm{L}, 2}(s)$ by minimizing the integral of absolute error (IAE). To assess the performance of disturbance rejection, the closed-loop transfer function from $L(s)$ to $y(s)$ according to eq 12 can be represented as

$$
\frac{y(s)}{L(s)}=\frac{g_{\mathrm{L}}(s)}{1+g_{\mathrm{C}} g(s)}=g_{\mathrm{L}}(s)[1-h(s)]
$$

The process in an MVC system can be decoupled; ${ }^{7,8}$ the resulting load transfer function is unchanged in the presence of such a decoupler. So, a multivariable system can be regarded as several independent single-loop systems, that respectively include the process $q_{i}(s)$ and disturbance $g_{\mathrm{L}, i}(s)$. Similarly, in MLC systems, a multivariable system can be decomposed into several equivalent single-loop systems as shown in eqs 5 and 6. In theory, to formulate $\bar{g}_{\mathrm{L}, i}(s)$ requires the controllers in all of the other loops. However, a method to circumvent this difficulty was given by Huang et al. ${ }^{10}$ In other words, $\bar{q}_{i}(s)$ and $\bar{g}_{\mathrm{L}, i}(s)$ can be given as 


$$
\begin{gathered}
\bar{q}_{i} \approx g_{i i}-G_{12}^{(i)}\left[G_{22}^{(i)}\right]^{-1} G_{21}^{(i)} \otimes \hat{H}^{(i)} \\
\bar{g}_{\mathrm{L}, i} \approx g_{\mathrm{L}, i}-G_{12}^{(i)}\left[G_{22}^{(i)}\right]^{-1} G_{L, 2}^{(i)} \otimes \hat{H}^{(i)}
\end{gathered}
$$

where $\hat{H}^{(i)}$ is a column vector consisting of $\hat{h}_{j}(s), j=1, \ldots, n$, and $i \neq j$. Notice that $\hat{h}_{j}(s)$ denotes an approximation of $h_{j}(s)$, which is specified to achieve reasonable control performance. The notation $\otimes$ means the element-to-element product of the two vectors.

So, the performances of disturbance rejection for MLC and DMVC systems are given by the following transfer functions:

$\begin{aligned} & \text { For MLC systems: } \frac{\bar{y}_{i}(s)}{L(s)}=\frac{\bar{g}_{\mathrm{L}, i}(s)}{1+g_{\mathrm{C}, i}(s) \bar{q}_{i}(s)}= \\ & \overline{\mathrm{g}}_{\mathrm{L}, i}(s)\left[1-w_{i, \mathrm{MLC}}(s)\right]\end{aligned}$

For DMVC systems: $\frac{y_{i}(s)}{L(s)}=\frac{g_{\mathrm{L}, i}(s)}{1+g_{\mathrm{C}, i}(s) q_{i}(s)}=$

$$
g_{\mathrm{L}, i}(s)\left[1-w_{i, \mathrm{MVC}}(s)\right]
$$

In other words, each of the systems has its complementary sensitivity function, $w_{i, \bullet}(s)$, that is $w_{i, \mathrm{MLC}}(s)$ or $w_{i, \mathrm{MVC}}(s)$, defined for the equivalent SISO loops. In practical design, usually, $w_{i, \bullet^{-}}$ $(s)$ needs to be confined to some simpler forms so that the performance can be specified clearly; and, the resulting controllers can be synthesized with reasonable costs. The optimal performance achievable under these restrictions is considered as the practically achievable performance. For this reason, the achievable performance depends on the structural constraints and the restrictions imposed on the design.

The two complementary sensitivity functions in eqs 16 and 17 can be expressed as the following:

$$
w_{i, \mathbf{\bullet}}(s)=w_{i, \bullet}^{o}(s) e^{-\theta_{a i, \bullet} s}
$$

where $w_{i, \boldsymbol{\bullet}}^{o}(s)$ is the delay-free part of $w_{i, \bullet}(s)$, and $\theta_{\mathrm{a}, i, \boldsymbol{\bullet}}$ is an apparent dead time, which is denoted as $\bar{\theta}_{\mathrm{a}, i}$ in $\bar{q}_{i}$ or denoted as $\theta_{\mathrm{a}, i}$ in $q_{i}$. If we confine that both $\bar{q}_{i}$ and $q_{i}(s)$ are approximately represented by FOPDT models and the diagonal controllers are confined to have a PID form, $w_{i, \bullet}^{o}(s)$ can be considered to have the following forms:

$$
w_{i, \bullet}^{o}(s)=\frac{z_{1, i} s+1}{p_{2, i} s^{2}+p_{1, i} s+1}
$$

or

$$
w_{i, \bullet}^{o}(s)=\frac{z_{2, i} s^{2}+z_{1, i} s+1}{p_{2, i} s^{2}+p_{1, i} s+1}
$$

Notice that $w_{i, \bullet}^{o}(s)$ must contain all RHP zeros in $\bar{q}_{i}(s)$ or $q_{i}(s)$. These RHP zeros can be estimated from the frequency responses of $\bar{q}_{i}(s)$ or $q_{i}(s)$, and can be directly embedded into $w_{i}^{o}(s)$. In the meantime, the resulting SISO system should have a reasonable maximum peak (i.e., $M s_{i, \bullet}$ ) ranging from 1.2 to 2.0 to ensure stability robustness. ${ }^{25}$ By adjusting the parameters in $w_{i, \mathbf{e}}^{o}(s)$ to optimize the performance under the restrictions above-mentioned, the practically achievable performance can be computed. Notice that $M s_{i, \bullet}$ is defined as

$$
\begin{array}{r}
M s_{i, \bullet}=\max _{\omega}\left\{\left|1-w_{i, \bullet}^{o}(j \omega) e^{-j \theta_{a i, \bullet} \omega}\right|\right\} ; \text { where, } M s_{i, \bullet}= \\
M s_{i, \mathrm{MLC}} \text { or } M s_{i, \mathrm{MVC}}
\end{array}
$$

The optimal parameters of $w_{i, \bullet}^{o}(s)$ in eq 19 with different $M s_{i, \bullet}$ specifications for standard SISO processes have been computed on the basis of the IAE and the FOPDT disturbance models and are given elsewhere. ${ }^{8}$ In this article, for simplification and to relax the limitation of FOPDT disturbance models, $w_{i, \bullet}^{o}(s)$ in eqs 19 and 20 are found by minimizing the two-norm errors (e.g., $\left\|y_{i}\right\|_{2}$ and $\left\|\bar{y}_{i}\right\|_{2}$ ), as discussed in the next section.

Once $w_{i, \bullet}^{o}(s)$ has been specified, the controllers to achieve the above optimal performance can be synthesized straightforwardly as follows

For MLC systems: $g_{\mathrm{C}, i}=$

$$
\frac{w_{i, \mathrm{MLC}}^{\mathrm{o}}}{\bar{q}_{\mathrm{o}, i}\left(\tau_{\mathrm{f}, i} s+1\right)^{m}} \frac{\left(1+\bar{\theta}_{\mathrm{a}, i} s / 2\right)}{\left(1+\bar{\theta}_{\mathrm{a}, i} s / 2\right)-w_{i, \mathrm{MLC}}^{\mathrm{o}}\left(1-\bar{\theta}_{\mathrm{a}, i} s / 2\right)}
$$

For DMVC systems: $g_{\mathrm{C}, i}=$

$$
\frac{w_{i, \mathrm{MVC}}^{o}}{q_{\mathrm{o}, i}\left(\tau_{\mathrm{f}, i} s+1\right)^{m}} \frac{\left(1+\theta_{\mathrm{a}, i} s / 2\right)}{\left(1+\theta_{\mathrm{a}, i} s / 2\right)-w_{i, \mathrm{MVC}}^{o}\left(1-\theta_{\mathrm{a}, i} s / 2\right)}
$$

where $\tau_{\mathrm{f}, i}$ is the filter time constant and has a default value of $0.05 \bar{\theta}_{\mathrm{a}, i}$ in MLC and $0.05 \theta_{\mathrm{a}, i}$ in DMVC. Furthermore, $m$ is selected to make $g_{\mathrm{C}, i}$ proper.

On the basis of the closed-loop transfer function of the disturbance rejection as shown in eqs 16 and 17, the achievable performance of an MLC system depends on the load transfer function of eq 15 and the apparent dead times in the equivalent open-loop transfer function, $\bar{q}_{i}(s)$. Similarly, the performance of a DMVC system depends on the original $G_{\mathrm{L}}(s)$ in eq 1 and the apparent dead times in the decoupled open-loop process, $q_{i}(s)$. These apparent dead times in both control systems are required to assign $w_{i, \bullet}(s)$ in eq 18 , and their values are introduced in the following remarks.

Remarks. (1) For MLC systems, the apparent dead time in $i$ th loop, $\bar{\theta}_{a, i}$, is the time it takes for $u_{i}$ to start affecting $y_{i}$. In an MLC system, $u_{i}$ can affect $y_{i}$ through different paths. So, $\bar{\theta}_{\mathrm{a}, i}$ equals the shortest dead time among all of the paths from $u_{i}$ to $y_{i}$. Because the controllers generally contain no dead time, the analysis is only based on the dead times of $G(s)$. The apparent dead time $\bar{\theta}_{a, i}$ in an $n \times n$ system is given as

$\bar{\theta}_{a, i}=\min _{j, k, \cdots y, z} \underbrace{\{\theta_{i i},\left[\theta_{i j}+\theta_{j i}\right], \cdots, \overbrace{\left[\theta_{i j}+\cdots+\theta_{k j}+\theta_{j i}\right]}^{n-1}, \overbrace{\left[\theta_{i z}+\theta_{z y}+\cdots+\theta_{k j}+\theta_{j i}\right]}^{n}\}}_{n}$

$$
\forall i, j, k, \ldots, y, z \in[1,2, \ldots, n] \text { and } i \neq j \neq \ldots \neq y \neq z
$$

For example, $\bar{\theta}_{\mathrm{a}, i}$ in a $2 \times 2$ system is

$$
\bar{\theta}_{\mathrm{a}, i}=\min _{j}\left\{\theta_{i i},\left[\theta_{i j}+\theta_{j i}\right]\right\} \forall i, j \in[1,2] \text { and } i \neq j
$$

and $3 \times 3$ system is

$$
\begin{aligned}
\bar{\theta}_{\mathrm{a}, i}=\min _{j, k}\left\{\theta_{i i},\left[\theta_{i j}+\theta_{j i}\right],\left[\theta_{i k}+\theta_{k j}+\theta_{j i}\right]\right\} \forall i, j, k \in \\
{[1,2,3] \text { and } i \neq j \neq k }
\end{aligned}
$$

(2) For DMVC systems, if there is no dead time in $\left|G_{0}(s)\right|$, the apparent dead time in DMVC systems can be easily taken as the dead times from $\Theta(s)$ in eq 7. However, factorization in eq 7 may cause $\left|G_{0}(s)\right|$ to have extra dead time in addition to $\Theta$ $(s)$. Notice that every $n \times n$ transfer-function matrix, $G(s)$, can always be factorized into the following form:

$$
G(s)=e^{-\Theta s} G_{0}(s)=e^{-\Theta s} \tilde{G}(s) e^{-\Lambda s}
$$


where $\Lambda=\operatorname{diag}\left\{\lambda_{1}, \cdots, \lambda_{n}\right\}$ and $\lambda_{i}$ is the dead time of the element in $G_{\mathrm{o}}(s)$. Notice that the determinant of $\tilde{G}(s)$ (i.e., $\left.|\tilde{G}(s)|\right)$ contains at least one term, which is free of dead time.

With the factorization in eq 25 , the extra dead time is taken as

$$
\theta_{\mathrm{ext}}=\sum_{i} \lambda_{i}
$$

So, the apparent dead time in DMVC systems is given by

$$
\theta_{\mathrm{a}, i}=\theta_{\mathrm{ext}}+\theta_{i i}^{*}
$$

and

$$
\Theta_{\mathrm{a}}=\left[\begin{array}{ll}
\theta_{\mathrm{a}, 1} & \\
& \ddots \\
& \theta_{\mathrm{a}, n}
\end{array}\right]=\Theta+\sum_{i} \lambda_{i}
$$

Thus,

$$
Q(s)=|\tilde{\mathrm{G}}(s)| \cdot e^{-\Theta_{a} s} \cdot Z(s)
$$

With this estimated apparent dead time, a practically achievable performance for the DMVC system can be computed by using eq 17.

For example, consider the TFM in eq 11, where, $G(s)$ can be written as

$$
G(s)=\left[\begin{array}{ll}
e^{-5 s} & 0 \\
0 & e^{-5 s}
\end{array}\right]\left[\begin{array}{ll}
\frac{9}{10 s+1} & \frac{0.8}{20 s+1} \\
\frac{0.9}{10 s+1} & \frac{8}{20 s+1}
\end{array}\right]\left[\begin{array}{ll}
1 & 0 \\
0 & e^{-5 s}
\end{array}\right]
$$

The $|\tilde{G}(s)|$ in the above factorization is free of dead time, but, $\left|G_{\mathrm{o}}(s)\right|$ is not. From eq 26, extra dead time is found in this case and is given as

$$
\theta_{\text {ext }}=5
$$

Then, by eq 28, the apparent dead times in case 2 are given as

$$
\Theta_{a}=\left[\begin{array}{ll}
10 & 0 \\
0 & 10
\end{array}\right]
$$

As shown in Figure 5, this extra dead time leads the system to a worse result in the first output of the DMVC system.

\section{Control Structure Assessment}

In the previous sections, estimations of practically achievable performances have been presented. On the basis of these performances, the two types of controllers can be assessed on the basis of the available information (e.g., steady-state gain matrix or TFM) about the process.

Assess by Steady-State Gains. As a measure of the change of manipulation responding to a load input, Stanley et al. ${ }^{26}$ proposed a relative disturbance gain (RDG) as an interaction measure. The RDG is formulated on the basis of steady-state gains of process and load and is formulated on an MLC basis. Skogestad and Morari ${ }^{27}$ introduced the disturbance condition number (DCN) to quantify the effect of the disturbance direction on closed-loop performance. In their method, the controller in the system needs to be given in detail. As a result, the DCN could not be obtained because the controller is not available. The extension of this RDG to a generalized disturbance gain
(GRDG) for the same purpose was given by Chang and Yu. ${ }^{28}$ Although applicable to the DMVC systems, this GRDG emphasizes differentiation if the interactions favor the change of the controller output but not the performance.

Theoretically, errors caused by the disturbances can only be eliminated after a dead-time period. During this period, the error magnitude in the output is proportional to the load gain of the system. Consequently, similar to the above-mentioned RDG, a relative load gain (RLG) is thus defined as follows.

$$
\phi_{i}=\frac{\left(\frac{\partial y_{i}}{\partial l}\right)_{\text {all loops except } i \text { closed }}}{\left(\frac{\partial y_{i}}{\partial l}\right)_{\text {all loops open }}}
$$

From this definition, RLG can be computed as

$$
\phi_{i}=\frac{\bar{g}_{\mathrm{L}, i}(0)}{g_{\mathrm{L}, i}(0)}=\frac{\bar{k}_{\mathrm{L}, i}}{k_{\mathrm{L}, i}}
$$

where $\bar{k}_{\mathrm{L}, i}$ and $k_{\mathrm{L}, i}$ are the gains of $\bar{g}_{\mathrm{L}, i}(s)$ and $g_{\mathrm{L}, i}(s)$, respectively. For example, in a $2 \times 2$ system, the RLG is obtained by computing the following:

$$
\begin{aligned}
& \phi_{1}=\frac{k_{\mathrm{L}, 1}-k_{12} k_{22}{ }^{-1} k_{\mathrm{L}, 2}}{k_{\mathrm{L}, 1}}=1-\frac{k_{12} k_{\mathrm{L}, 2}}{k_{22} k_{\mathrm{L}, 1}} \\
& \phi_{2}=\frac{k_{\mathrm{L}, 2}-k_{21} k_{11}{ }^{-1} k_{\mathrm{L}, 1}}{k_{\mathrm{L}, 2}}=1-\frac{k_{21} k_{\mathrm{L}, 1}}{k_{11} k_{\mathrm{L}, 2}}
\end{aligned}
$$

Notice that the above $\phi_{i}$ equals $\beta_{i} / \lambda_{i i}$, where $\lambda$ and $\beta$ are the RGA and RDG, respectively. According to the definitions, $\beta_{i}$ $<1$ or a larger $\lambda>1$ implies that interactions are in favor of control. The expression in eq 35 shows how these quantities are related to the performance in a steady-state sense. We defined the absolute value of RLG as an index

$$
\epsilon(K)=\frac{\sum_{i} \alpha_{i}\left|\phi_{i}\right|}{\sum_{i} \alpha_{i}} \text { and } \epsilon_{i}(K)=\left|\phi_{i}\right|
$$

where $\epsilon(K)$ designates a lumped index for assessing MLC against DMVC based on steady-state gains; $\epsilon_{i}(K)$ designates the similar index on an individual-loop basis. When $\epsilon(K)<1$, it means that an MLC system may have an overall performance better than that of a DMVC system. On the other hand, for any loop that has $\epsilon_{i}(K)<1$, the loop interaction favors that output so that a SISO controller may be sufficient for that particular loop. By making use of eq 36 in case 1 , one can see that the point $\left(k_{\mathrm{L}, 1}, k_{\mathrm{L}, 2}\right)$ lies in the dark and gray areas in Figure 6, which implies $\epsilon_{1}(K)<1$ and $\epsilon_{2}(K)>1$, respectively. For example, when $k_{\mathrm{L}, 1}=5$ and $k_{\mathrm{L}, 2}=4, \epsilon_{1}(K)=0.6$ and $\epsilon_{2}(K)=$ 0.29 ; and when $k_{\mathrm{L}, 1}=5$ and $k_{\mathrm{L}, 2}=-4, \epsilon_{1}(K)=1.4$ and $\epsilon_{2}(K)=1.71$. These results with their implications agree well with simulation results, as have been observed previously in Section 2.

Although the presented index in terms of RLG is useful, it is not consistent in a dynamic simulation. This is because it is based on steady-state gains and does not take the dynamic effects into account. More-consistent results can be obtained, if this index can take the process dynamics into account. In the 
(a)

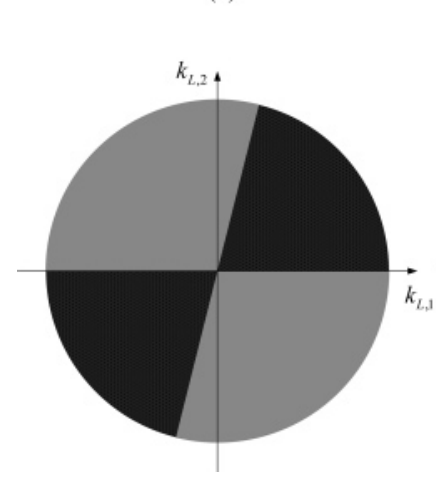

(b)

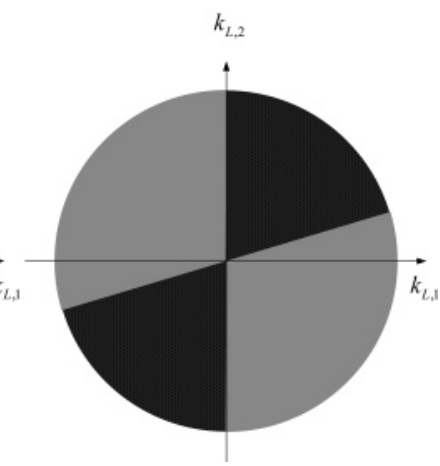

Figure 6. Assessment for case 1 based on steady-state RLG indices, (a) the first loop and (b) the second loop.

following, a criterion that considers dynamics of the process will be proposed.

Assessment by Dynamic TFM. In the previous section, the indices for assessing the two control structures are based on an assumption that only steady-state gains are available. A more reliable index is to use information not only from the steady state gain but also from its dynamics.

For MLC Systems. To estimate the control performance for MLC systems, eq 16 is used. The approximate equivalent disturbance transfer function is given in eq 15, where each element in the matrix $\hat{H}^{(i)}$ is given by eqs 19 or 20 , whose parameters have been optimized with respect to $g_{i i}(s)$ and $g_{\mathrm{L}, i^{-}}$ $(s)$. The estimation of the optimal performance in MLC systems is to minimize the following two-norm error by adjusting the parameters in $w_{i, \mathrm{MLC}}$.

$$
\left\|\overline{\mathrm{y}}_{i}\right\|_{2}=\sqrt{\frac{1}{2 \pi} \int_{-\omega_{\mathrm{f}}}^{\omega_{\mathrm{f}}}\left|\overline{\mathrm{g}}_{\mathrm{L}, i}(j \omega)\left[1-w_{i, \mathrm{MLC}}(j \omega)\right] L(j \omega)\right|^{2} d \omega}
$$

Here, $\omega_{\mathrm{f}}$ is the frequency bandwidth of concern. The value of $\omega_{\mathrm{f}}$ has to be selected large enough beyond the bandwidth of transfer function $\bar{q}_{i}$. In this work, $\omega_{\mathrm{f}}$ is taken as 10 times the bandwidth or greater.

For DMVC Systems. The responses of disturbance rejection in DMVC systems can be treated as the responses in singleloop systems as given in eq 17 , where $w_{i, \operatorname{MVC}}(s)$ is the complementary sensitivity function for each decoupled system. The two-norm error for practical disturbance rejection of DMVC systems can be obtained by minimizing the following with $w_{i, \mathrm{MVC}}$.

$\left\|y_{i}\right\|_{2}=\sqrt{\frac{1}{2 \pi} \int_{-\omega_{\mathrm{f}}}^{\omega_{\mathrm{f}}}\left|g_{\mathrm{L}, i}(j \omega)\left[1-w_{i, \mathrm{MVC}}(j \omega)\right] L(j \omega)\right|^{2} d \omega}$

Then, on the basis of dynamics, an index to assess the performance of MLC against DMVC is defined as

$$
\epsilon(G)=\frac{\sum_{i} \alpha_{i}\left\|\bar{y}_{i}\right\|_{2}\left\|y_{i}\right\|_{2}^{-1}}{\sum_{i} \alpha_{i}} \text { and } \epsilon_{i}(G)=\frac{\left\|\bar{y}_{i}\right\|_{2}}{\left\|y_{i}\right\|_{2}}
$$

When the index $\epsilon(G)$ is less than one, it means the overall performance of MLC is better than that of DMVC. Similarly, $\epsilon_{i}(G)<1$ means the interaction favors the performance of the ith loop.

Remark. As mentioned, the individual criteria $\epsilon_{i}(K)$ and $\epsilon_{i^{-}}$ $(G)$ provide implications that if interactions in an MIMO system favor or don't favor the control of individual output $y_{i}$. As a result, the assessment can be concluded as

$$
\begin{cases}\varepsilon_{i}(K)>1 \text { or } \varepsilon_{i}(G)>1 ; & \text { Interactions in MLC system do not favor output } y_{i} \\ \varepsilon_{i}(K)<1 \text { or } \varepsilon_{i}(G)<1 ; & \text { Interactions in MLC system facvor output } y_{i}\end{cases}
$$

In the case where $\epsilon_{i}>1$ for some $i$ and $\epsilon_{i}<1$ for the others, it implies that interactions favor some outputs in an MLC system. Thus, a full decoupling will not benefit the whole system. As a result, a partial decoupling controller may have to be considered to achieve better performance.

Examples. Example 1. Consider the Wood and Berry process. ${ }^{29}$ In the nominal situation, the process and load transferfunction matrices are given as follows

$$
G(s)=\left[\begin{array}{cc}
\frac{12.8 e^{-s}}{16.7 s+1} & \frac{-18.9 e^{-3 s}}{21 s+1} \\
\frac{6.6 e^{-7 s}}{10.9 s+1} & \frac{-19.4 e^{-3 s}}{14.4 s+1}
\end{array}\right]
$$

and

$$
G_{\mathrm{L}}(s)=\left[\begin{array}{c}
\frac{3.8 e^{-8.1 s}}{14.9 s+1} \\
\frac{4.9 e^{-3.4 s}}{13.2 s+1}
\end{array}\right]
$$

First, steady-state gains are used to compute the assessing indices for the nominal case. From eq 34, the RLGs calculated are: $\phi_{1}=-0.26$ and $\phi_{2}=0.6$. From eq 36 , it gives $\epsilon_{1}(K)=$ 0.26 and $\epsilon_{2}(K)=0.6$.

To take into account the dynamics, the indices will be computed under $M s_{1}=M s_{2}=1.6$. First, in MLC systems, $\hat{h}_{i}$ used in eq 15 are found as follows:

$$
\begin{aligned}
\hat{h}_{1}=\frac{1.157 s^{2}+2.117 s+1}{2.247 s^{2}+1.358 s+1} e^{-s} \text { and } \hat{h}_{2}= \\
\qquad \frac{7.739 s^{2}+4.469 s+1}{14.45 s^{2}+3.231 s+1} e^{-3 s}
\end{aligned}
$$

So, the equivalent disturbances can be found by eq 15 . According to eq 24, the apparent dead times can be determined as $\bar{\theta}_{\mathrm{a}, 1}=1$ and $\bar{\theta}_{\mathrm{a}, 2}=3$. Then, the two-norm errors for disturbance input are found by minimizing eq 37 . Second, in DMVC systems, the apparent dead times in both loops are determined by eq 28 as 1 and 3, respectively. Similarly, the two-norm error for disturbance input in DMVC systems are found by minimizing eq 38 . Finally, the assessing indices in eq 39 can be obtained as $\epsilon_{1}(G)=0.557$ and $\epsilon_{2}(G)=1.08$ for the nominal case. Notice that the indices based on steady-state gains are not consistent with those based on dynamics.

Next, to validate the assessment, controllers are designed and their performances are examined by simulation. Assume that the robust specifications are to have $M s_{1}=M s_{2}=1.6$. In MLC, the sequential method is applied for designing the PID controllers. The controller parameters are found iteratively by minimizing the two-norm errors. So, the resulting controllers are given as

$$
\begin{aligned}
g_{\mathrm{C}, 1}=\frac{0.313 s^{2}+0.686 s+0.171}{s(0.05 s+1)} \text { and } g_{\mathrm{C}, 2}= \\
\frac{-0.223 s^{2}-0.0892 s-0.0319}{s(0.15 s+1)}
\end{aligned}
$$


(a)
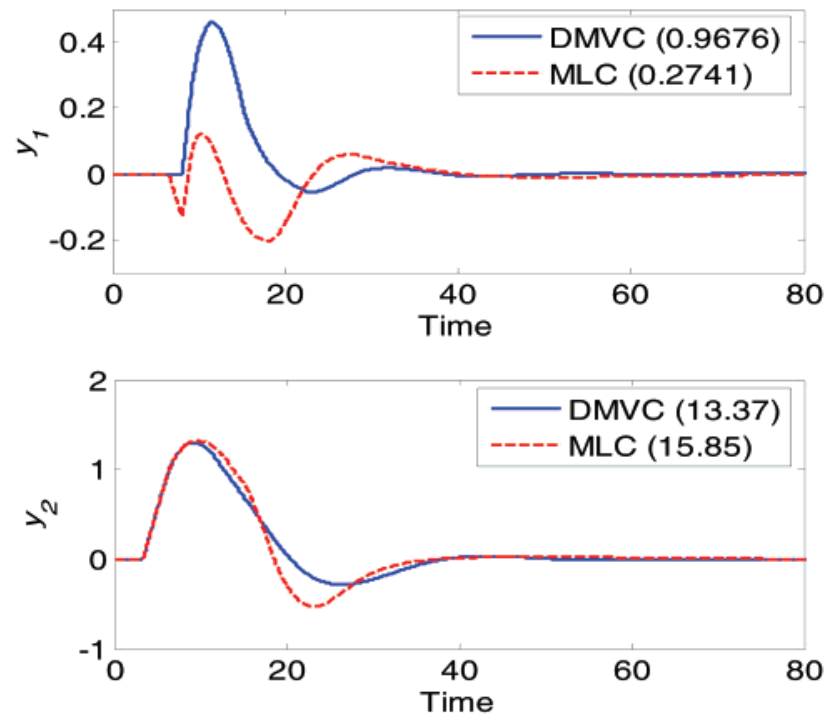

(b)
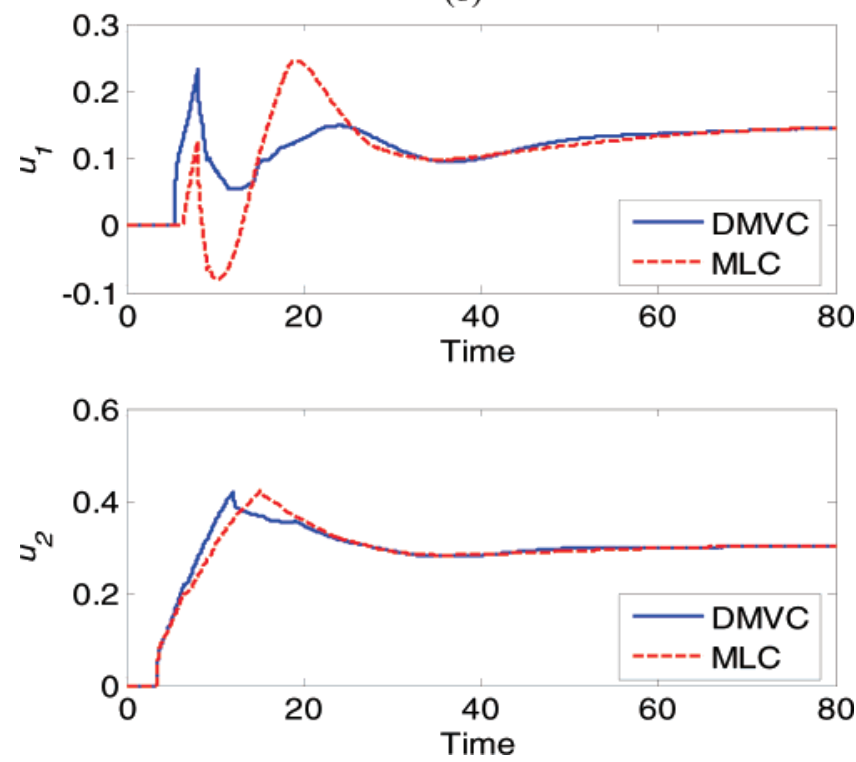

Figure 7. (a) Responses and (b) control signals of MLC and DMVC for the Wood and Berry process.

In DMVC control, the decoupler is designed by the method of Huang and $\operatorname{Lin}^{8}$ so that $q_{1}$ and $q_{2}$ are obtained as

$$
q_{1}=\frac{-123.58 e^{-s}}{14.13 s+1} \text { and } q_{2}=\frac{-123.58 e^{-3 s}}{14.13 s+1}
$$

Then, the optimal PID controllers are designed for each decoupled loop to minimize the two-norm errors to obtain

$$
C_{1}=\frac{-0.0216 s^{2}-0.0435 s-0.0121}{s(0.05 s+1)}
$$

and

$$
C_{2}=\frac{-0.0316 s^{2}-0.0211 s-0.00384}{s(0.15 s+1)}
$$

The simulated responses, their individual ISE values, and control signals to a unit-step disturbance input are shown in Figure 7. From the results, in the nominal case, MLC shows better performance in the first loop but slightly worse perfor- (a)
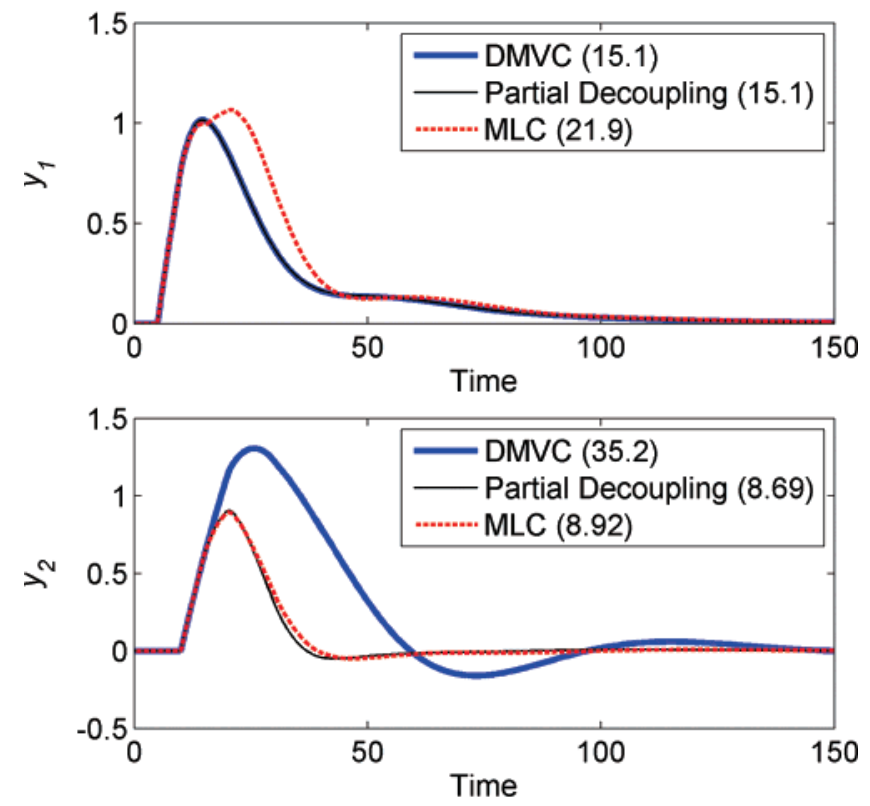

(b)
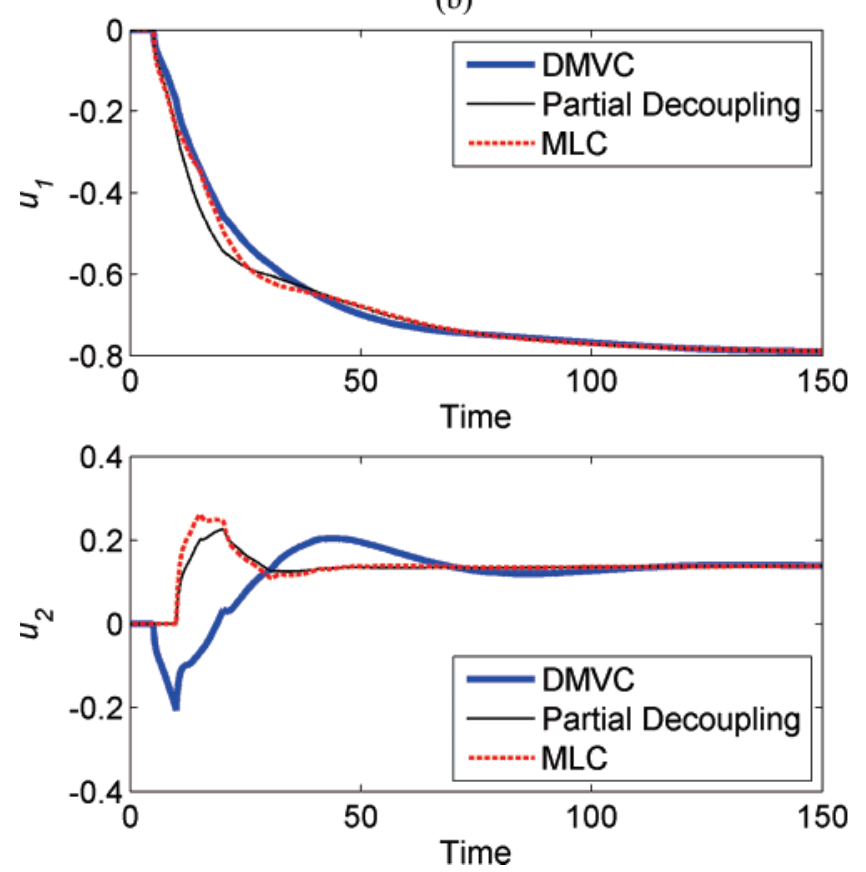

Figure 8. (a) Responses and (b) control signals of MLC, DMVC, and partial decoupling for example 2.

mance in the second loop. This agrees with the result from the computed indices.

Example 2. In this example, a partially decoupled system is used to illustrate the effectiveness of the results implied by $\epsilon_{i}$. Consider a system that contains the following transfer-function matrices:

$$
G(s)=\left[\begin{array}{cc}
\frac{7 e^{-5 s}}{10 s+1} & \frac{4 e^{-5 s}}{20 s+1} \\
\frac{4 e^{-10 s}}{10 s+1} & \frac{-6 e^{-10 s}}{20 s+1}
\end{array}\right]
$$




$$
G_{\mathrm{L}}=\left[\begin{array}{c}
\frac{5 e^{-5 s}}{30 s+1} \\
\frac{4 e^{-10 s}}{30 s+1}
\end{array}\right]
$$

First, $G(s)$ is factorized into

$$
G=\left[\begin{array}{ll}
e^{-5 s} & 0 \\
0 & e^{-10 s}
\end{array}\right]\left[\begin{array}{ll}
\frac{7}{10 s+1} & \frac{4}{20 s+1} \\
\frac{4}{10 s+1} & \frac{-6}{20 s+1}
\end{array}\right]
$$

From eq 34, RLGs are computed in the following:

$$
\phi_{1}=1.53 \text { and } \phi_{2}=0.29
$$

Assume that the control system is required to have $M s_{1}=M s_{2}$ $=1.6$. The proposed indices give $\epsilon_{1}(K)=1.53, \epsilon_{2}(K)=0.29$, $\epsilon_{1}(G)=1.24$, and $\epsilon_{2}(G)=0.25$. These indices imply that DMVC is better to control the first output, but MLC is better for the second output.

Similarly, validation with simulations is performed in the following. For this purpose, optimal PID controllers for MLC controls are designed and given as

$$
g_{\mathrm{C}, 1}=\frac{0.4351 s^{2}+0.1896 s+0.02469}{s(0.25 s+1)}
$$

and

$$
g_{\mathrm{C}, 2}=\frac{-1.292 s^{2}-0.1774 s-0.01087}{s(0.5 s+1)}
$$

Then, the DMVC controller is also designed by the method of Huang and Lin. ${ }^{8}$ The resulting open-loop processes and optimal PID controllers are determined as

$$
\begin{gathered}
q_{1}=\frac{-58 e^{-5 s}}{10 s+1} \\
q_{2}=\frac{-58 e^{-10 s}}{20 s+1} \\
C_{1}=\frac{-0.0437 s^{2}-0.02335 s-0.003296}{s(0.25 s+1)} \\
C_{2}=\frac{-0.1216 s^{2}-0.02433 s-0.002052}{s(0.5 s+1)}
\end{gathered}
$$

A partial decoupler is also designed for this MIMO system, that is

$$
D=\left[\begin{array}{cc}
1 & \frac{-4(10 s+1)}{20 s+1} \\
0 & 7
\end{array}\right]
$$

and

$$
Q=\left[\begin{array}{ll}
\frac{-7 e^{-5 s}}{10 s+1} & 0 \\
\frac{4 e^{-10 s}}{10 s+1} & \frac{-58 e^{-10 s}}{20 s+1}
\end{array}\right]
$$

Optimal PID controllers are also obtained in the following:

$$
\begin{gathered}
C_{1}=\frac{0.363 s^{2}+0.1945 s+0.02725}{s(0.25 s+1)} \\
C_{2}=\frac{-0.1158 s^{2}-0.02357 s-0.001588}{s(0.5 s+1)}
\end{gathered}
$$

The output responses and control signals in three controls together with their individual ISE values in responding to a unitstep disturbance input are all shown in Figure 8. Comparing the results with those mentioned above, the proposed indices indeed suggest a correct control structure.

\section{Conclusion}

Multiloop and decoupling multivariable controllers are commonly used in the control of the MIMO processes. Prior to the design of the controller, selecting a proper type of controller is an important issue. For these purposes, methods to assess the performance between two types of systems are proposed. These methods are derived from the practically achievable performances of the two systems, without resorting to their controllers in details. The first method makes use of the RLG to compute both the overall and the individual indices based on the steadystate gain matrix of the process only. Similar to the case of RGA, this method does not always give consistent results in all cases. This is because the indices are lacking effects from the dynamics of the process. The second method uses two norms of achievable dynamic responses to derive either overall or individual indices. Illustrations of the use of the proposed method to select between MLC and DMVC are also given. The results show that the proposed indices are effective and useful.

\section{Acknowledgment}

The work is supported by the National Science of Council of Taiwan (NSC-93-2214-E-002-008).

\section{Literature Cited}

(1) Niederlinski, A. Two-Variable Distillation Control: Decouple or Not Decouple. AIChE J. 1971, 17, 1261.

(2) Harris, T. J.; Boudreau, F.; MacGregor, J. F. Performance Assessment of Multivariable Feedback Controllers. Automatica 1996, 32, 1505.

(3) Harris, T. J.; Seppala, C. T.; Desborough, L. D. Review of Performance Monitoring and Assessment Techniques for Univariate and Multivariate Control Systems. J. Process Control 1999, 9, 1.

(4) Huang, B.; Shah, S. L.; Kwok, E. K. Good, Bad or Optimal? Performance Assessment of Multivariable Processes. Automatica 1997, 33, 1175.

(5) Huang, B; Shah, S. L. Performance Assessment of Control Loops; Springer-Verlag: London, 1999.

(6) Agamennoni, O. E.; Desages, A. C.; Romagnoli, J. A. Robust Controller Design Methodology for Multivariable Chemical Processes. Chem. Eng. Sci. 1988, 43, 2937.

(7) Wang, Q. G.; Zhang, Y.; Chiu, M. S. Non-Interacting Control Design for Multivariable Industrial Processes. J. Process Control 2003, 13, 253.

(8) Huang, H. P.; Lin, F. Y. Decoupling Multivariable Control with Two Degrees of Freedom. Ind. Eng. Chem. Res. 2006, 45, 3161.

(9) Leithead, W. E.; O'Reilly, J. Performance Issues in the Individual Channel Design of 2-Input 2-Output Systems. Int. J. Control 1991, 54, 47.

(10) Huang, H. P.; Jeng, J. C.; Chiang, C. H.; Pan, W. A Direct Method for Multi-Loop PI/PID Controller Design. J. Process Control 2003, 13, 769.

(11) Luyben, W. L. Simple Method for Tuning SISO Controllers in Multivariable Systems. Ind. Eng. Chem. Process Des. Dev. 1986, 25, 654

(12) Monica, T. J.; Yu, C. C.; Luyben, W. L. Improved Multiloop SingleInput/Single-Output (SISO) Controllers for Multivariable Processes. Ind. Eng. Chem. Res. 1988, 27, 969.

(13) Mayne, D. Q. The Design of Linear Multivariable Systems. Automatica 1973, 9, 201.

(14) Chiu, M. S.; Arkun, Y. A Methodology for Sequential Design of Robust Decentrialized Control Systems. Automatica 1992, 28, 997. 
(15) Hovd, M.; Skogestad, S. Sequential Design of Decentrialized Controllers. Automatica 1994, 30, 1601.

(16) Shiu, S. J.; Hwang, S. H. Sequential Design Method for Multivariable Decoupling and Multiloop PID Controllers. Ind. Eng. Chem. Res. 1998, 37, 107

(17) Shen, S. H.; Yu, C. C. Use of Relay-Feedback Test for Automatic Tuning of Multivariable Systems. AIChE J. 1994, 40, 627.

(18) Lee, J.; Cho, W.; Edgar, T. F. Multiloop PI Controller Tuning for Interacting Multivariable Processes. Comput. Chem. Eng. 1998, 22, 1711.

(19) Wang, Q. G.; Lee, T. H.; Zhang, Y. Multi-Loop Version of The Modified Ziegler-Nichols Method for Two Input Two Output Process. Ind. Eng. Chem. Res. 1998, 38, 4725.

(20) Bao, J.; Forbes, J. F.; McLellan, P. J. Robust Multiloop PID Controller Design: A Successive Semidefinite Programming Approach. Ind. Eng. Chem. Res. 1999, 38, 3407.

(21) Economou, C. G.; Morari, M. Internal Model Control: 6. Multiloop Design. Ind. Eng. Chem. Process Des. Dev. 1986, 25, 411.

(22) Grosdidier, P.; Morari, M. Interaction Measures for Systems under Decentralized Control. Automatica 1986, 25, 309.

(23) Skogestad, S.; Morari, M. Robust Performance of Decentralized Control Systems by Independent Designs. Automatica 1989, 25, 119.
(24) Hovd, M.; Skogestad, S. Improved Independent Design of Robust Decentrialized Controllers. J. Process Control 1993, 3, 43.

(25) Chen, D.; Seborg, D. E. PI/PID Controller Design Based on Direct Synthesis and Disturbance Rejection. Ind. Eng. Chem. Res. 2002, 41, 4807.

(26) Stanley, G.; Marino-Galarraga, M.; McAvoy, T. J. Short-Cut Operability Analysis: 1. The Relative Disturbance Gain. Ind. Eng. Chem. Process Des. Dev. 1985, 24, 1181.

(27) Skogestad, S.; Morari, M. Effect of Disturbance Directions on Closed-Loop Performance. Ind. Eng. Chem. Res. 1987, 26, 2029.

(28) Chang, J. W.; Yu, C. C. Relative Disturbance Gain Array. AIChE J. 1992, 4, 521 .

(29) Wood, R. K.; Berry, M. W. Terminal Composition Control of a Binary Distillation Column. Chem. Eng. Sci. 1973, 28, 1707.

(30) Basualdo, M. S. Dynamic Interaction Compensators for Multiloop Control Structure. Chem. Eng. Commun. 1995, 134, 231.

Received for review March 14, 2007 Revised manuscript received September 27, 2007 Accepted September 28, 2007

IE070382I 